\title{
Improved assessment of vertebral cortex thickness by means of analytical deconvolution of radial bone mineral density distributions
}

T. Damm¹, J. Peña ${ }^{1}$, J. Bastgen ${ }^{1}$, S. Waldhausen ${ }^{1}$, G. Campbell1, R. Barkmann ${ }^{1}$, I. Frieling ${ }^{2}$, M. Krause ${ }^{3}$, C.-C. Glüer ${ }^{1}$

${ }^{1}$ Section Biomedical Imaging, Clinic of Radiology, University Hospital Schleswig-Holstein (UKSH), Campus Kiel, Germany

2Osteoporosezentrum Hamburg-Neuer Wall, Hamburg, Germany

${ }^{3}$ Institut für Osteologie und Biomechanik, Universitätsklinikum Hamburg-Eppendorf, Germany

Corresponding author's email: timo.damm@rad.uni-kiel.de

\section{Background}

Assessment of bone stability using computed tomography data requires - besides densitometric parameters - an accurate estimation of the cortical thickness, which in QCT and to a lesser extent also in HRQCT is biased by the partial volume effect and the CT systems modulation transfer function (MTF).

In HRQCT the spatial resolution is, due to an estimated gaussian point spread function (PSF) with a full-width at half-maximum (FWHM) of $(0,97 \pm 0,10) \mathrm{mm} \quad(\delta=0,41 \pm 0,04)$, significantly reduced compared to the actual voxel size and even compact structures are broadened, as can be seen in the figure on the right.

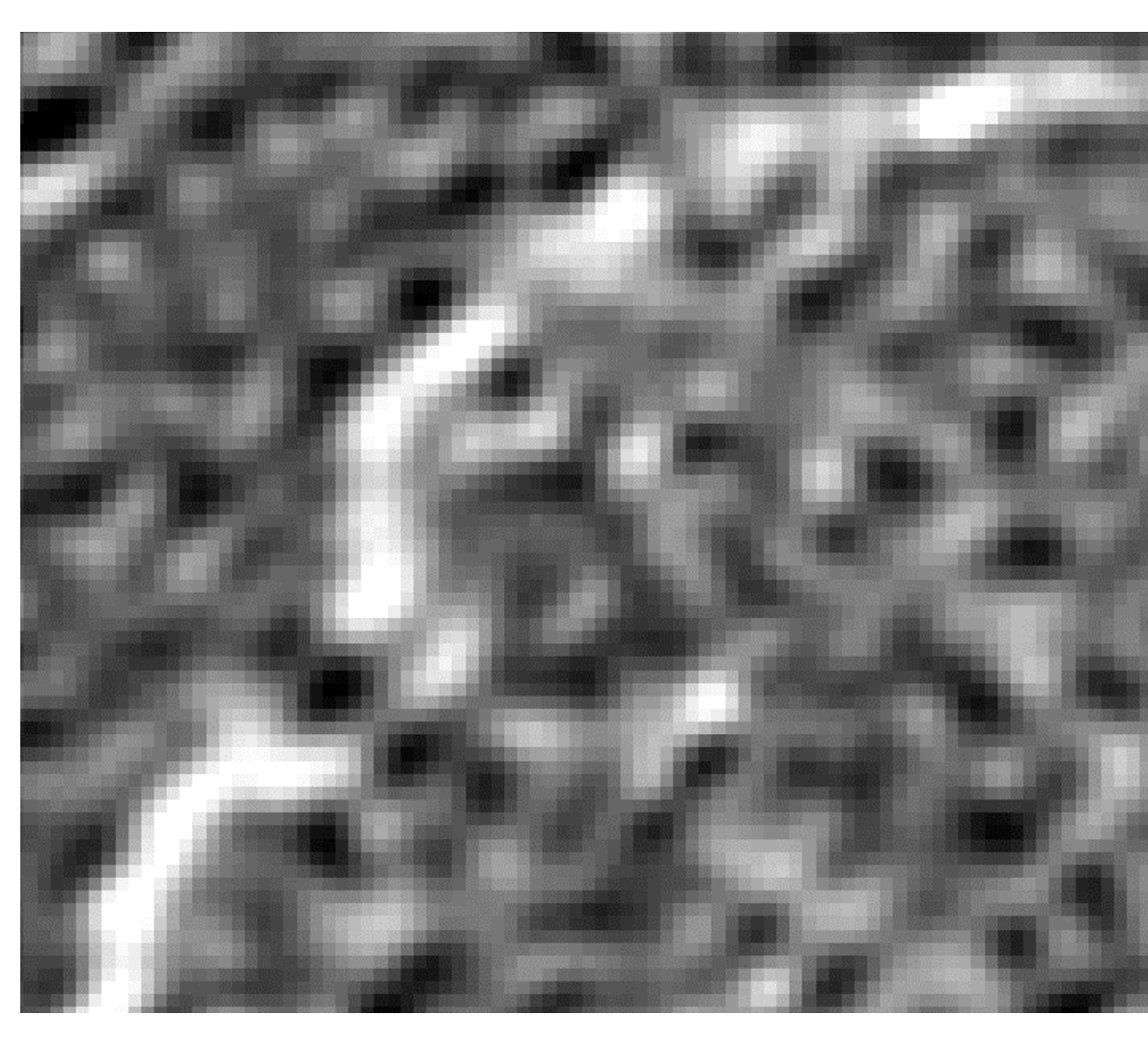

Materials and Methods

We analysed 12 excised human vertebrae embedded in epoxy compound. HRpQCT scans were carried out at the Osteoporosezentrum Hamburg and HRQCT scans at the University Hospital Schleswig-Holstein in Kiel. Image acquisition parameters are listed in table 1.

\begin{tabular}{lll}
\hline Table 1 & HRpQCT & HRQCT \\
\hline Device & Scanco Medical XtremeCT & Siemens Somatom 64 \\
Voxel Size in $\mathrm{mm}^{3}$ & $0,082^{*} 0,082^{*} 0,082$ & $0,156{ }^{*} 0,156{ }^{*} 0,300$ \\
Voltage in $\mathrm{kV}$ & 60 & 120 \\
Exposure in $\mathrm{mAs}$ & 190 & 360 \\
\hline Reconstruction Kernel & standard & B70s \\
\hline
\end{tabular}

Data Processing with Structural/nsight

1. Quality Assurance prior to data analysis

2. Calibration of Hunsfield Units (HU) to

$\mathrm{mgHA} / \mathrm{cc}$ using a simultaneously scanned phantom

3. Template-driven semi-automatic

segmentation of the different volumes of interest (VOI): total trabecular volume,

ellipse, vertical cortex, upper end plate, lower end plate, foramen and cut pedicles

At this point, the segmentation based cortical thickness Ct.Th and the density weighted cortical Thickness wCt.Th can be calculated.

The deconvolution-based refinement requires

two further processing steps:

1. Generation of layers at both sides of the inner segmentation border of the vertical cortex with a constant thickness (e.g. $0.2 \mathrm{~mm}$ ) each

2. Calculations of mean bone mineral densities (BMD) within these equidistant layers

This $\mathrm{BMD}$ (ridge distance) data is used in a

forward modelling approach as outlined in the

next paragraph. Fig. 3: Trabecular network (beige) covered by the 3D

layers to generate mean

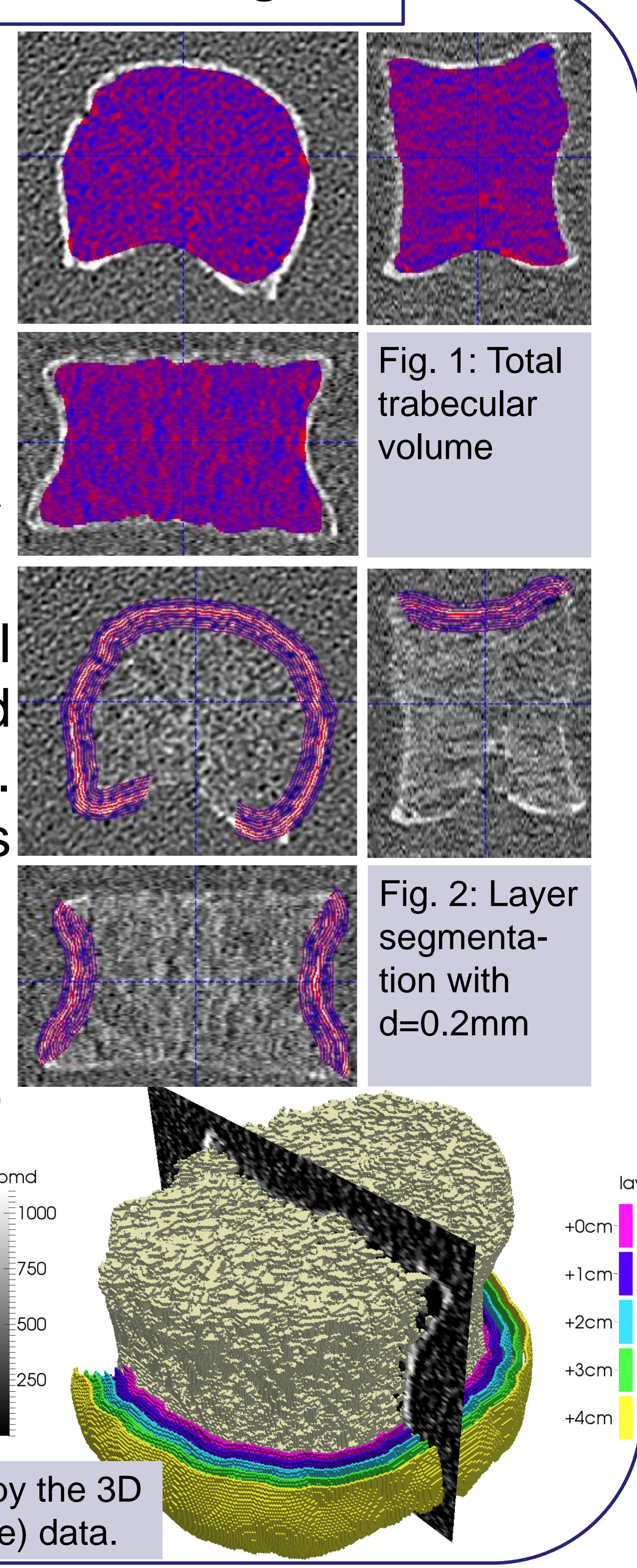

Deconvolution-based Forward Model

- Assuming an gaussian distribution for the MTF and accordingly for the PSF of the CT imaging system

- Deducing an edge-spread-function (ESF) and a

non-infesitesimal line-spread-function (LSF)

- Putting the ESF for the spongiosa plateau and the LSF for the cortex into one function

- Setting the full mineralization to $1100 \mathrm{mgHA} / \mathrm{cc}$

- Fitting spongiosa plateau (ESF) and cortex plateau (LSF) on the BMD(ridge distance) data yields the deconvoluted cortical thickness dcCt.Th"

Fitting an additional epoxy plateau outside the bone (yellow) results in dcCt.Th*

This fit procedure can be done with a free $\delta$, actually turning the cortex itself into a "test body" to access the PSF's FWHM.

\section{Results}

The diagrams below show the $\mathrm{BMD}$ (ridge distance) data points and the approximation by the forward model (using the epoxy compound term) as a line for all 10 vertebrae, resulting in solutions for $\mathrm{dcCt}$.Th*.

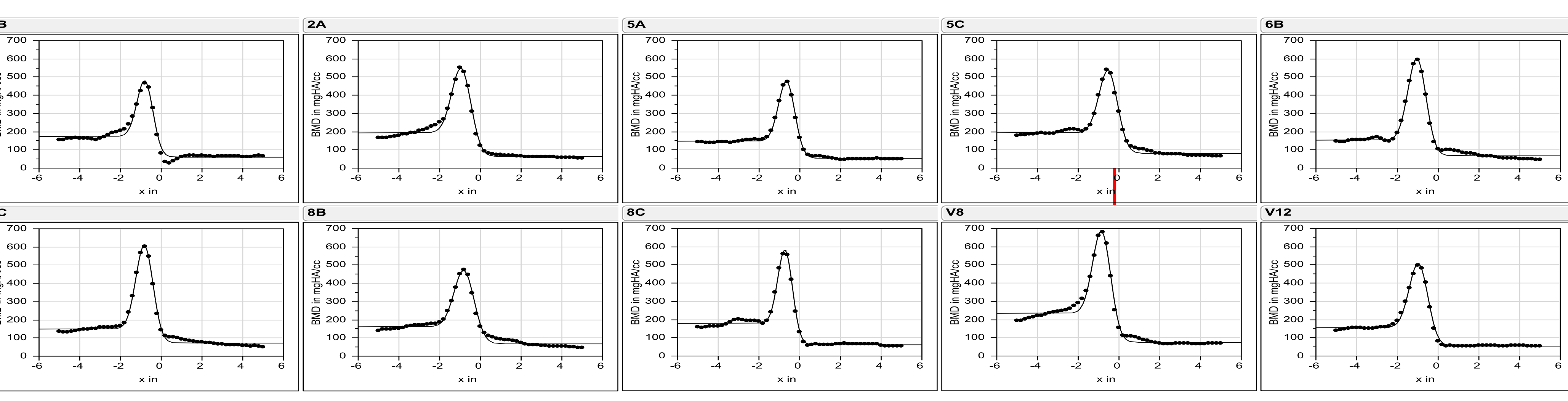

For HRpQCT scans, we find a mean cortical thickness of $(0,36 \pm 0,07)$ $\mathrm{mm}$ using Scanco Medical software (DT-Tb.Th within the cortex segmentation). Using Structual/nsight on HRQCT data, the mean thickness of the vertical cortex segmentations is $(1.52 \pm 0.27) \mathrm{mm}$. Using the deconvolution-based forward modeling and assuming a mineralization of $1100 \mathrm{mgHA} / \mathrm{cc}$, a mean of $(0.46 \pm 0.08) \mathrm{mm}$ was obtained (table 2).

\begin{tabular}{|c|c|c|c|c|c|}
\hline Table 2 & HRPQCT & \multicolumn{4}{|c|}{ HRQCT } \\
\hline & DT Ct.Th & Ct.Th & wCt.Th & dcCt.Th & dcCt.Th* \\
\hline Mean Cortical Thickness in $\mathrm{mm}$ & $0.36 \pm 0.07$ & $1.52 \pm 0.27$ & $0.52 \pm 0.13$ & $0.49 \pm 0.09$ & $0.46 \pm$ \\
\hline RMSE (HRQCT against HRpQCT) in mm & & 0.058 & 0.037 & 0.019 & 0.019 \\
\hline $\mathrm{r}^{2}$ (HRQCT against HRpQCT) & & 0.38 & 0.75 & 0.93 & 0.93 \\
\hline
\end{tabular}

Graph 1 shows a weak correlation between HRQCT Ct.Th and the HRPQCT DT-Ct.Th

Graph 1 RMSE $=0.058 \mathrm{~mm}$.

This is contrasted by the strong correlation between the deconvolution-based HRQCT dcCt.Th ${ }^{*}$ and the HRpQCT DT-Ct.Th $\left(r^{2}=0.93, R M S E=0.019 \mathrm{~mm}\right)$ in graph 2 .

Graph 3 shows the Bland-Altman plot for them, which better illustrates the residual errors with a mean offset of $(0.096 \pm 0.021) \mathrm{mm}$ without a significant slope.

\section{Graph 3}

In the 3D visualization on the right, the vertical cortex (clipped for clarification) is shown with dcCt. Th $^{*}=0.43 \mathrm{~mm}$ from HRQCT as an example. In HRpQCT, this vertebra's DT-Ct.Th was $0.35 \mathrm{~mm}$.

Fig. 5: Estimated cortex (clipped) over a 2D slice of BMD as assessed by HRQC

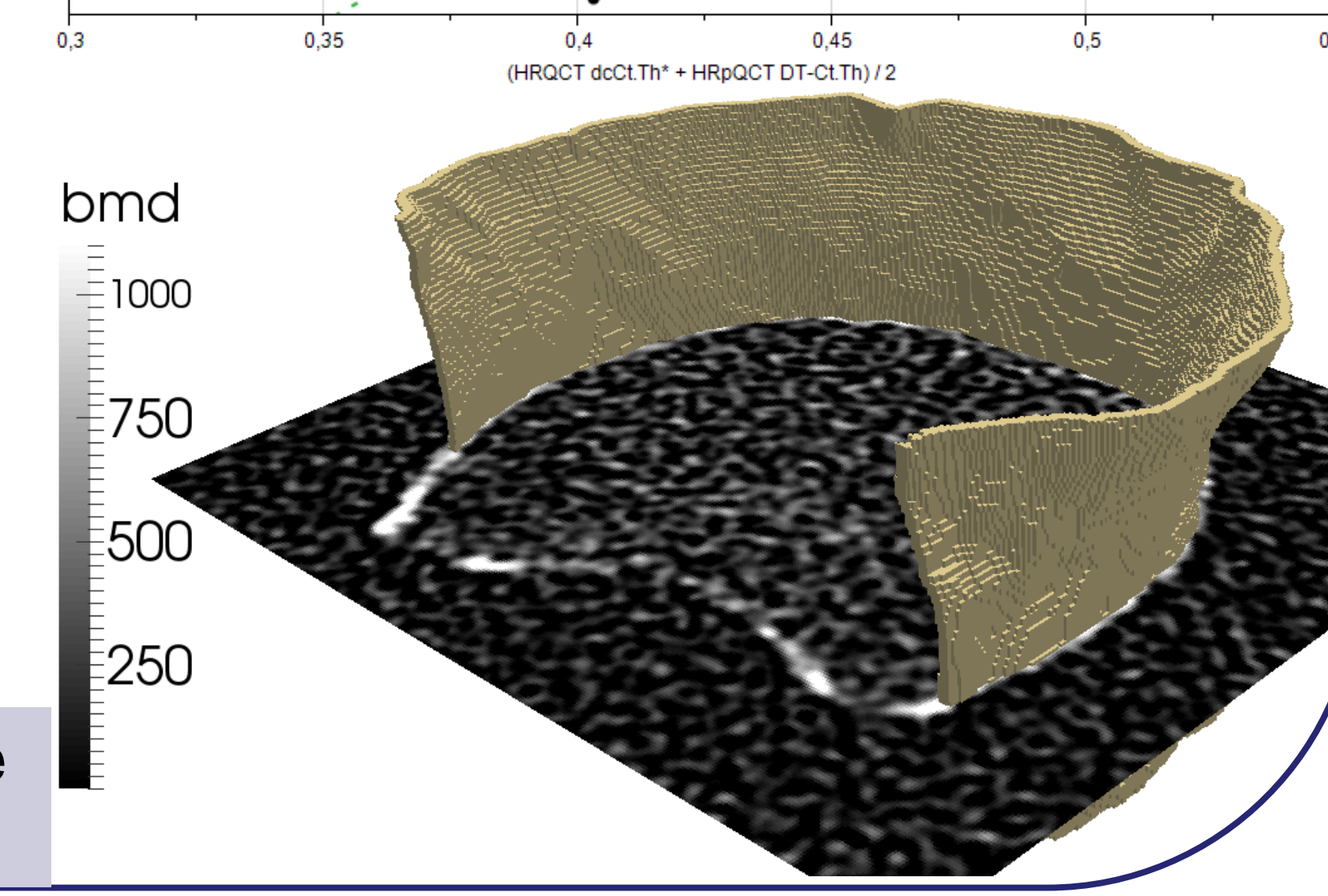

\section{Conclusion}

Deconvolution-based correction reduced the accuracy error of cortical thickness (dcCt.Th*) measurements obtained on HRQCT data to $28 \%$ (compared to goldstandard HR-pQCT) whereas accuracy error of segmentation-based $\mathrm{Ct}$.Th and its density-weighted refinement wCt.Th had levels of $423 \%$ and $45 \%$, respectively.

Using sufficient thick layers, noise is reduced and the use of the forward model in itself is another source of stability.

The cortex can be used as a test body to estimate the scanner- and kernel-dependent point spread function.

After semi-automatic segmentation, the determination of the deconvoluted cortical thickness runs without user interaction and, except for the mineralization, without further adjustable parameters. 